\title{
High-speed Droplet Impingement in Compressible Regime
}

\author{
Mason Marzbali, Ali Dolatabadi \\ ${ }^{1}$ Department of Mechanical, Industrial and Aerospace Engineering, Concordia University \\ 1455 De Maisonneuve Blvd. W., Montreal, QC, H3G 1M8, Canada \\ mason.marzbali@concordia.ca; ali.dolatabadi@concordia.ca
}

\begin{abstract}
High-speed droplet impact is of great interest to power generation and aerospace industries due to the accrued cost of maintenance in steam and gas turbines. The repetitive impacts of liquid droplets onto rotor blades, at high relative velocities, result in the blade erosion, which is known as Liquid Impingement Erosion (LIE). Experimental and analytical studies in this field are limited due to the complexity of the droplet impact at such conditions. Hence, numerical analysis is a very powerful and affordable tool to investigate LIE phenomenon. In this regard, it is crucial to understand the hydrodynamics of the impact in order to identify the consequent solid response before addressing the LIE problem. The numerical study of the droplet impingement allows to obtain the transient pressure history generated in the liquid. Knowing the transient behavior of the substrate, in response to pressure force exerted due to the impact, would facilitate engineering new types of surface coatings that are more resistant to LIE. To that end, modeling the impact of compressible liquid droplets at high velocities on rigid solid substrates is the main objective of the present work. The results obtained from the compressible fluid modeling are validated against the numerical studies and analytical correlations, available in open literature.
\end{abstract}

Keywords: Liquid impingement erosion, compressible droplet impact, CFD, VOF.

\section{Introduction}

Although the experimental study of droplet impacts dates back to 1876 by great scientists such as Worthington [1], it still remains an area of extensive study today. The advancement of high-speed imaging techniques, in addition to computational power, have allowed researchers to obtain much higher temporal and spatial resolutions in capturing the hydrodynamics of droplet impingement. There are various physical phenomena that occur upon the impact of a liquid droplet on a solid substrate, such as spreading, fingering, air entrapment, bouncing and splashing, that drew the attention of various industries and academia. For instance, the spreading of a droplet at low impingement velocities has been studied in details by Fukai et al. [2], Chandra and Avedisian [3], Pasandideh-Fard et al. [4] and more recently by Roisman et al. [5]. The entrapment of an air bubble underneath the droplet has been simulated by Mehdi-Nejad et al. [6]. Moreover, the formation of fingers upon impact of water drops was experimentally investigated by Mehdizadeh et al. [7]. Blake and De Conick [8] have extended the molecular-kinetic theory of dynamic wetting by considering the effect of fluid-solid interaction. A 2-D axisymmetric, two-way coupled Fluid-Solid Interaction model for impingement of liquid droplets onto rigid and elastic Ti6Al-4V substrate was presented by Marzbali [9] and Marzbali et al. [10]. It was illustrated that the fluid compressibility effect on the pressure build-up and solid stress cannot be neglected for impingement velocities of $100 \mathrm{~m} / \mathrm{s}$ and above. However, most of the past studies in droplet impingement are concerned with low impact velocities, hence, do not take into account the fluid compressibility, which becomes critical at high impingement velocities.

Upon the impact of a liquid droplet on a solid substrate at a high velocity, compression waves begin to propagate in the water droplet due to the fluid compressibility. These compression waves create a high density region in the liquid close to the solid surface and break away from the droplet periphery, giving rise to the formation of lateral jets, as reported by Heymann [11] and confirmed by the experiments of Dear et al. [12] and Field et al. [13]. The pressure waves generated in the liquid accumulate over time and form a high-pressure front since the speed of sound changes in the compressed region. Compression waves travel through the compressed region at the speed of sound in the liquid, hence, the rest of the droplet, outside the disturbed region, is not affected by the impact yet. The pressure and density inside the undisturbed region remain unchanged since it is completely unaware of the impact. As the liquid droplet continues its downward motion toward the solid, the high-pressure front penetrates further into the liquid bulk and the compressed region increases in size. At the same 
time, the droplet contact line is moving radially outward from the droplet center axis, right after the droplet touches down on the surface.

Initially, the contact periphery spreads out much faster than the high-pressure front. As the contact line starts to decelerate due to viscous dissipation, it is finally overtaken by the high-pressure front that is spreading outward. At this exact instant, the high-pressure front detaches and starts to travel along the free surface leading to the formation of lateral jets. This jetting eruption is the result of a very large difference between the pressure and density across the free surface of the droplet in contact line region. When lateral jetting occurs, the impact pressure reaches its temporal maximum value, as experimentally measured for the first time by Rochester and Brunton [14]. It has been shown by Heymann [11] that the pressure in the compressed area is not uniform and the location where the highest pressure appears is behind the contact line.

Lesser and Field [15] studied the droplet impact analytically and in order to determine the exact position of the highpressure front, an envelope of individual wavelets emitted by the expanding contact edge was proposed. In this model, it was assumed that the compression wave velocity is equal to the speed of sound in ambient condition. Although this assumption is valid for the acoustic limit, Haller [16] demonstrated that it is invalid for high impact velocities where the liquid compressibility effect is considerable. He has shown, both computationally and analytically, that the compression wave velocity during the first stage of the impact, at an impingement velocity of $500 \mathrm{~m} / \mathrm{s}$, is in the range of 2600-3000 $\mathrm{m} / \mathrm{s}$, which is significantly higher than the ambient speed of sound in water, i.e. $1430 \mathrm{~m} / \mathrm{s}$. Therefore, in modeling the compressible droplet impact, it is essential to account for the changes in the speed of sound, via the variation of liquid density, with an equation of state. In this regard, the main objective of the current work is to study the effect of liquid compressibility on the generated pressure build-up via high-speed droplet impingements. To that end, a compressible Volume of Fluid (VOF) model is utilized to resolve the interfacial gas-liquid flow. The obtained results for lateral jet velocity, liquid density and maximum pressure are compared with the study reported by Haller [16].

\section{Methodology}

\subsection{Governing equations}

Navier-Stokes equations are solved for two compressible and immiscible fluids. The conservation of mass and momentum equations in their transient and compressible forms are as follows,

$$
\begin{gathered}
\frac{\partial \rho_{f}}{\partial t}+\nabla \cdot\left(\rho_{f} V_{f}\right)=0 \\
\frac{D\left(\rho_{f} V_{f}\right)}{D t}=\nabla \cdot \sigma_{f}+\rho_{f} F_{b}
\end{gathered}
$$

where $V_{f}$ is the fluid velocity vector, $\rho_{f}$ is the fluid density, $F_{b}$ is body forces acting on the fluid. $\sigma_{f}$ is the Cauchy stress tensor for a Newtonian fluid, including pressure and viscous terms, defined as,

$$
\sigma_{f}=-p_{f} I+\tau_{f}
$$

where $p_{f}$ is the fluid pressure, $I$ is the 3 by 3 identity matrix and $\tau_{f}$ is the viscous stress tensor,

$$
\tau_{f}=\mu_{f}\left(\nabla V_{f}+\nabla V_{f}^{T}\right)-\frac{2}{3} \mu_{f}\left(\nabla \cdot V_{f}\right) I
$$

where $\mu_{f}$ is the fluid dynamic viscosity. A single momentum equation is solved for both gas and liquid phases and fluid properties are calculated for the mixture. The fluid flow is assumed to be laminar; hence, no turbulence model is employed in the solver. The energy equation for a compressible flow is as follows, 


$$
\frac{D\left(\rho_{f}\left[e+V_{f}^{2} / 2\right]\right)}{D t}+\nabla \cdot\left(V_{f} p_{f}\right)=-\nabla \cdot q+\nabla \cdot\left(\tau_{f}\right) \cdot V_{f}+\rho_{f} g \cdot V_{f}
$$

where $e=c T$ is the internal energy and $q$ is the heat flux vector, calculated based on the gradient of the internal energy,

$$
q=-\alpha \nabla e
$$

where $\alpha$ is the thermal diffusivity. In addition, in order to close the system of equations, an equation of state for gas and liquid phases is needed. The ideal gas law is applied to account for the air compressibility as follows,

$$
p_{g}=\rho_{g} R T_{g}
$$

where $R$ is the specific gas constant equal to $287 \mathrm{~J} / \mathrm{kgK}$ for air. For the liquid phase, the power law equation of state proposed by Tait [17], commonly used for water, is utilized and defined below,

$$
\frac{p_{l}+B}{p_{a}+B}=\left(\frac{\rho_{l}}{\rho_{l_{0}}}\right)^{N}
$$

where $\rho_{l_{0}}$ is the density of water at ambient conditions equal to $1000 \mathrm{~kg} / \mathrm{m}^{3}$ and $B, p_{a}$ and $N$ are three constants from Tait's equation equal to $B=300 M P a, p_{a}=0.1 M P a$ and $N=7.415$ [17], respectively.

The surface tension force is treated as a pressure gradient across the liquid-gas interface and is calculated per unit volume based on the Continuum Surface Force (CSF) model proposed by Brackbill et al. [18]. The compressible VOF model adapted from Hirt and Nichols [19] is employed to resolve the droplet interface. In VOF method, a scalar field is defined for volume fraction of the liquid phase, namely $\alpha$, and its value depends on the fraction of the cell volume occupied by this phase. The values between zero and one denote the interface between gas and liquid phases. Since the volume fraction represents the volume occupied by the liquid, it should be advected by the flow field at each time step. The following equation governs the liquid advection,

$$
\frac{\partial\left(\rho_{f} \alpha\right)}{\partial t}+\nabla \cdot\left(\rho_{f} \alpha V_{f}\right)=0
$$

Following the advection, the interface is reconstructed using the Piecewise Linear Interface Calculation (PLIC) proposed by Youngs [20]. In PLIC method, the interface is defined at each computational cell by a slope and an intercept. The slope of the interface is calculated based on the volume fractions of the neighboring cells.

\subsection{Computational domain, boundary and initial conditions}

The computational domain for the 2-D axisymmetric model has a width of eight times the droplet radius $(R)$ and the height of the domain is $4 R$. The gravitational force is exerted in the same direction as the droplet impingement. The computational domain consists of air and water phases and the properties at ambient conditions are utilized for both phases. Outflow boundary condition is applied to all fluid boundaries except for the substrate surface. No-slip condition is imposed on the fluid-solid interface. In addition, the droplet center axis has axisymmetric boundary condition.

Initially, the fluid domain is filled with air. At the beginning of each simulation the droplet is patched in the fluid domain with the desired size and velocity. The computation for the rest of the cells starts with zero for the velocity and liquid volume fraction. The initial temperature and pressure in all the computational cells including the boundaries are set to the ambient conditions, i.e. $300 \mathrm{~K}$ and $100 \mathrm{kPa}$, respectively.

\subsection{Numerical schemes and discretization}

The Pressure-Implicit with Splitting of Operators (PISO) method is used for pressure-velocity coupling in transient calculations. The integral forms of fluid equations are discretized over each control volume and solved for a fixed system of 
grids in a segregated manner. The solution is then obtained by using a time marching scheme based on the given initial condition. The Gaussian integration method is used for summation of the values on cell faces interpolated linearly from centers. The time scheme is first-order implicit based on Euler and is bounded. The gradient schemes are Gauss linear with central differencing. The divergence schemes are also Gaussian with an upwind discretization for all the transport equations except for volume fraction flux which is second-order accurate in space and unbounded. Finally, the uncorrected Gauss linear scheme is utilized in discretizing the Laplacian equation. For other variables, such as temperature, density and kinetic energy, an upwind method, which is first order and bounded, is used to discretize the equations. The second derivative of temperature is discretized with a linear scheme, which is second order and unbounded. The linear Gaussian scheme is utilized for interpolation from the cell centers to the faces.

The time step during for all the advective fluxes in transport equations is different from the one used in VOF calculations. The time step in VOF is adaptive and depends on the Courant-Friedrichs-Lewy (CFL) condition. The maximum CFL is set to 0.1 during the computations to constrain the adaptive time step. This requirement ensures that the liquid advection through one cell is less than $10 \%$ at every time step and the initial time step is determined based on the impact velocity and grid size, depending on the impact condition. Finally, OpenFoam, an open-source computational code, is used for programming the solver. In OpenFoam, all the equations are solved in three dimensions and a 2-D axisymmetric mesh is simply a $5^{\circ}$ section of a full cylinder with flat sides and one cell in lateral direction, which results in a wedge. In presenting some of the results, the variables are nondimensionalized with respect to the following reference values: droplet radius $(R)$, impact velocity $\left(V_{0}\right)$, water hammer pressure $\left(p_{w h}\right)$, density of undisturbed water $\left(\rho_{0}\right.$ $\left.=1000 \mathrm{~kg} / \mathrm{m}^{3}\right)$ and initial temperature $\left(T_{0}=300 \mathrm{~K}\right)$. As a convention, “*” denotes a dimensionless variable.

\subsection{Grid sensitivity analysis}

The dependence of the generated pressure on the grid size is investigated. To that end, the impact of a 500- $\mu \mathrm{m}$ droplet at $350 \mathrm{~m} / \mathrm{s}$ on a rigid substrate is analyzed with three different grid sizes of $1.25,1$ and $0.75 \mu \mathrm{m}$. The peak dimensionless pressures predicted with the grid size of $1.25,1$ and $0.75 \mu \mathrm{m}$ are $2.2925,2.2583$ and 2.2527, respectively. The difference of the peak pressure values between the simulation with 1 and $0.75 \mu \mathrm{m}$ grids is less than $0.25 \%$. Therefore, refining the grid size below $1 \mu \mathrm{m}$ would not affect the accuracy of the generated pressure and this mesh size is selected for simulating the droplet impact.

\section{Results and discussion}

The results obtained for a droplet diameter of $200 \mu \mathrm{m}$ and an impact velocity of $500 \mathrm{~m} / \mathrm{s}$ are reported. The dimensionless values over nine time steps for pressure, velocity, density and temperature are plotted in Fig. 1, Fig. 2, Fig. 3 and Fig. 4, (a) and (b) along radial and axial directions, respectively. As it can be seen in Fig. 1 (a) and (b), the pressure starts to build up right after the droplet touches down on the solid surface. In addition, a distinct region is observed where the pressure is orders of magnitude higher than the rest of the droplet, indicating the compressed region, which is separated from the undisturbed area with the high-pressure front mentioned earlier. Moreover, a stagnation region is formed upon the impact. At $t^{*}=0.005$ after the impact the pressure reaches the water hammer value $\left(\mathrm{p}^{*}=1\right)$ at the center $\left(\mathrm{r}^{*}=0\right)$. However, at $\mathrm{r}^{*}=0.114$ from the center, $\mathrm{p}^{*}$ reaches a value of 2.2 , suggesting that the maximum pressure occurs off the droplet axis with a magnitude more than twice the water hammer pressure.

As it can be seen in Fig. 1 (b), the pressure is not maximum on the surface but slightly above it along the y-axis at $y^{*}=0.0267$. This is to be expected, as the liquid region closer to the high-pressure front experiences a higher pressure compared to the rest of the compressed region. The main reason behind this phenomenon is the fact that each fluid particle that comes in contact with the solid surface emits a compression wave that moves in the opposite direction of the impact. Since the liquid density increases after the passage of each compression wave, hence, the speed of sound is higher in this region, the consequent compression wave, emitted due to the impact of another fluid particle on the surface, would travel faster through the disturbed region. As a result, the compression waves that have been generated lately would eventually overtake the previously emitted waves, forming a strong pressure front where the gradients of fluid variable are very sharp. This can be seen in Fig. 2, where the vertical velocity is close to zero in a narrow depth, the compressed region, and jumps to the impact velocity with a steep slope. In fact, the fluid density outside the compressed region remain unchanged, as illustrated in Fig. 3, which means that the rest of the droplet is completely unaware of the impact. 

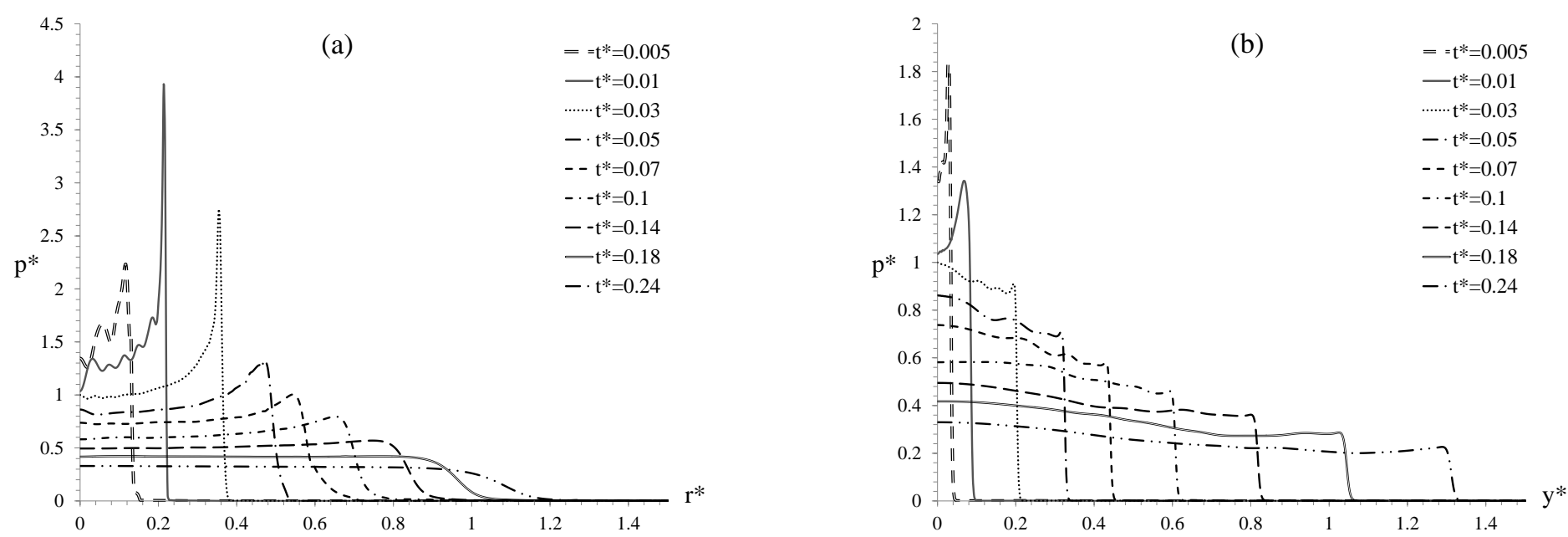

Fig. 1: Dimensionless pressure along (a) radial and (b) axial directions.
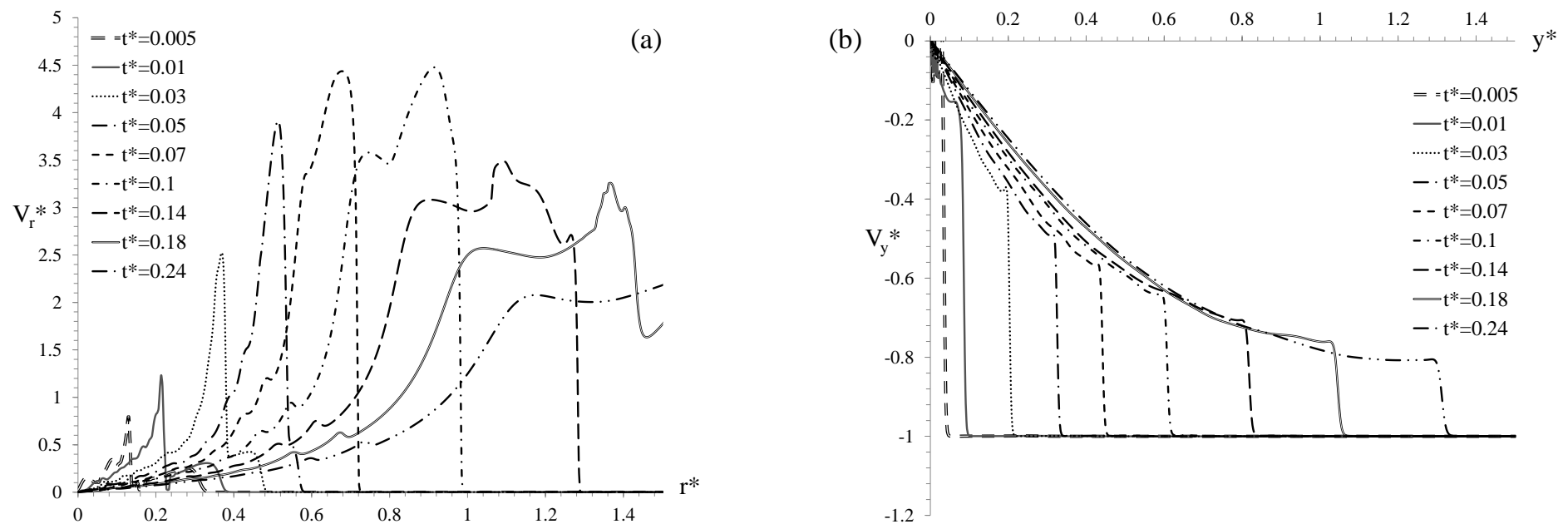

Fig. 2: Dimensionless (a) radial velocity and (b) axial velocity.

Theoretically, the contact line moves with an infinite velocity, however, the fluid particle velocity has a finite value and its radial component $\left(\mathrm{V}_{\mathrm{r}}{ }^{*}\right)$ is plotted for various time steps in Fig. 2 (a). The droplet edge velocity increases as the droplet spreads over the solid surface up to $t^{*}=0.07$ and then start to decelerate due to the viscous dissipation. Around this time step, the air is expelled from the droplet periphery after the eruption of the lateral jet, which explains the appearance of a double hump in the radial velocity. During the acceleration period, the high-pressure front moves behind the contact line and it remains attached to the interface. After the deceleration of the contact line begins, the high-pressure front overtakes the contact line. At this moment, the pressure front detaches from the droplet edge and lateral jetting begins. When the highpressure front reaches the droplet edge, it starts to move along the free surface. The reflection of the compression wave from the droplet interface creates an expansion wave normal to the free surface. The generated expansion waves rapidly reduce the pressure in the fluid particles located between the free surface and the high-pressure front. In other words, they neutralize the influence of the compression waves close to the free surface. As a result, this region experiences a lower pressure. The low-pressure region caused by this rarefaction could lead to cavitation inside the droplet as observed experimentally by Field et al. [13]. These rarefaction waves also prevent the lateral jetting from the droplet free surface by rapid pressure reduction. Therefore, lateral jetting only occurs on the droplet edge attached the fluid-solid interface, where the expansion waves are absent due to the presence of the solid surface. It should be noted that the pressure reaches its peak, $\mathrm{p}^{*}=3.93(2.8 \mathrm{GPa})$, at $\mathrm{t}^{*}=0.01$, which occurs before the high-pressure front overtakes the free surface, around $\mathrm{t}^{*}=0.03$, followed by its detachment at $\mathrm{t}^{*}=0.05$. 

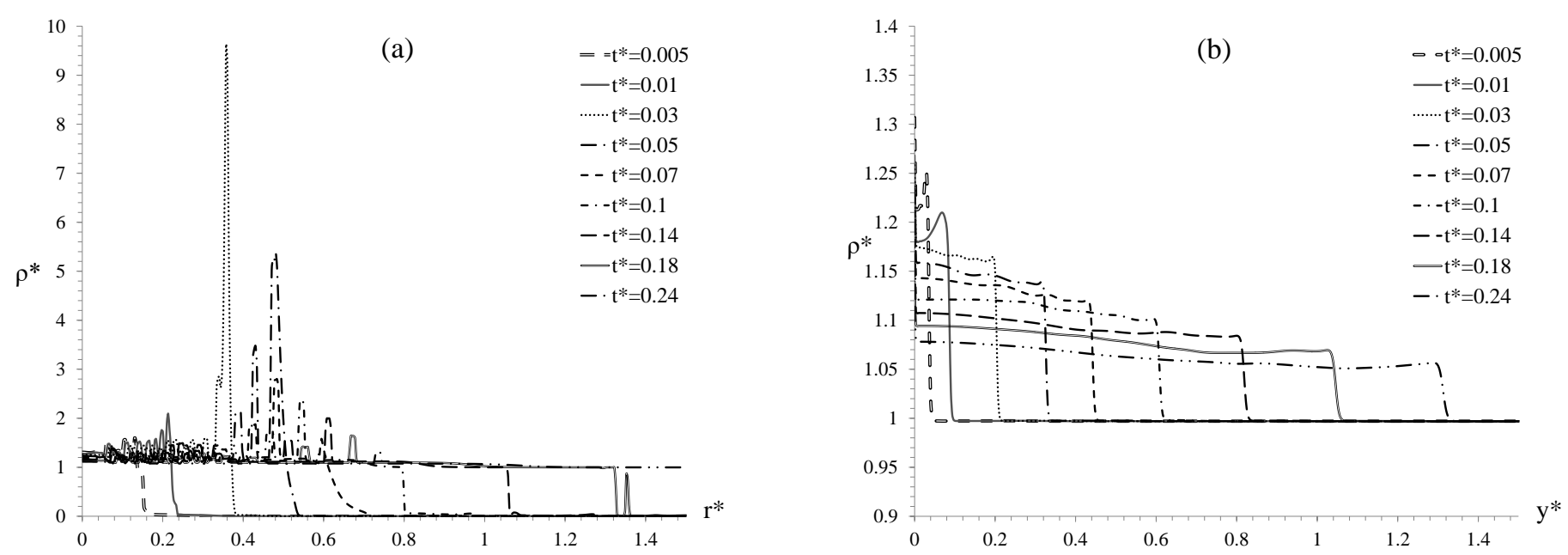

Fig. 3: Dimensionless density along (a) radial and (b) axial directions.
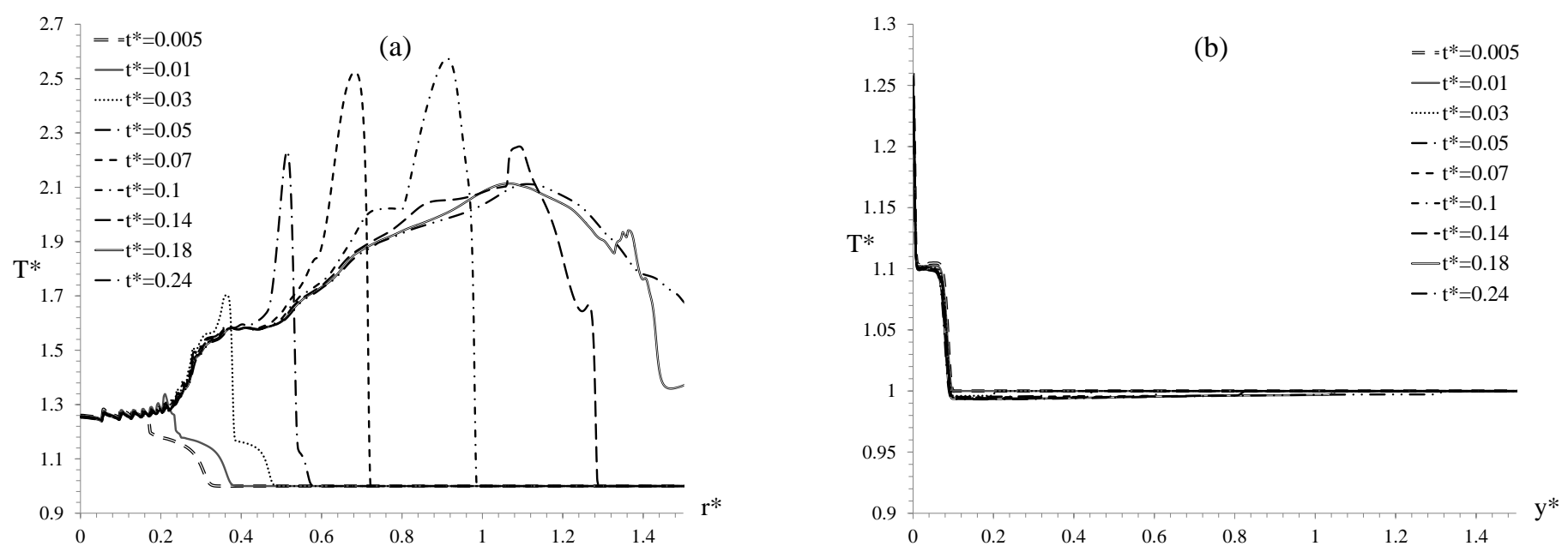

Fig. 4: Dimensionless temperature along (a) radial and (b) axial directions.

A close look at the density, plotted in Fig. 3, reveals that, at $t^{*}=0.03, \rho^{*}=9.61$ at a radial location of $\mathrm{r}^{*}=0.357$. Examining the liquid volume fraction for the cells located at $r^{*}=0.357$ suggest that they are filled mostly with air. Since the pressure at this point reaches a value of $\mathrm{p}^{*}=2.75(1.96 \mathrm{GPa})$ and the temperature is equal to $\mathrm{T}^{*}=1.7(510 \mathrm{~K})$, the ideal gas law would result in a similar value for the air density. The graphs of dimensionless temperature, presented in Fig. 4 , demonstrate that the temperature in the compressed region is around $\mathrm{T}^{*}=1.1$ along the droplet center axis with the maximum of $\mathrm{T}^{*}=1.25$ on the solid surface $\left(\mathrm{y}^{*}=0\right)$. More importantly, the temperature is much higher at the droplet periphery with a maximum of $\mathrm{T}^{*}=2.57$ at $\mathrm{t}^{*}=0.1$, which is right after the eruption of lateral jets. The droplet edge has the highest temperature compared to the rest of the liquid bulk since it has the highest kinetic energy (due to its velocity). The viscous dissipation in the fluid that is in contact with the solid surface, partially converts this kinetic energy to thermal energy, hence, the heat transfer in this region is enhanced. As a result, the lateral jet that is erupted from the droplet periphery reaches a temperature as high as $771 \mathrm{~K}\left(\mathrm{~T}^{*}=2.57\right)$. This is well above the boiling temperature of water $(373 \mathrm{~K})$, provided that the pressure at this radial location, i.e. $\mathrm{r}^{*}=0.9$, is atmospheric. Therefore, the water that is attached to the solid surface close to the droplet edge would evaporate when jet eruption occurs. This would lead to strong cavitation and a vapor rim would be created underneath the impinging droplet. It should be noted that the equations for the phase change are not integrated in the current model, hence, such a cavitation due to the water evaporation cannot be captured here.

Fig. 5 (a) and (b) illustrate the density and radial velocity after lateral jetting occurs, from the present work at $t^{*}=0.07$ and reported by Haller [16] after 3.05 ns, respectively. Both studies suggest that the droplet edge reaches a velocity of 
$2325 \mathrm{~m} / \mathrm{s}\left(\mathrm{V}_{\mathrm{r}}{ }^{*}=4.65\right)$, which is considerably higher than the impact velocity $(500 \mathrm{~m} / \mathrm{s})$. However, the maximum pressure observed in the compressed region inside the droplet is equal to $\mathrm{p}^{*}=3.93(2.8 \mathrm{GPa})$, which is $16 \%$ higher than the peak pressure reported by Haller [16], i.e. close to $2.4 \mathrm{GPa}$, for the same impact condition. This is to be expected since in his model, Haller [16] utilized the stiffened gas equation of state for water resulting in a linear relation, whereas, in Tait's equation of state, the pressure-density relation is a power-law with an exponent of 7.415. Furthermore, the time of the lateral jetting and the location where it occurs are different in the two studies due to neglecting the viscous force in Haller's model [16].
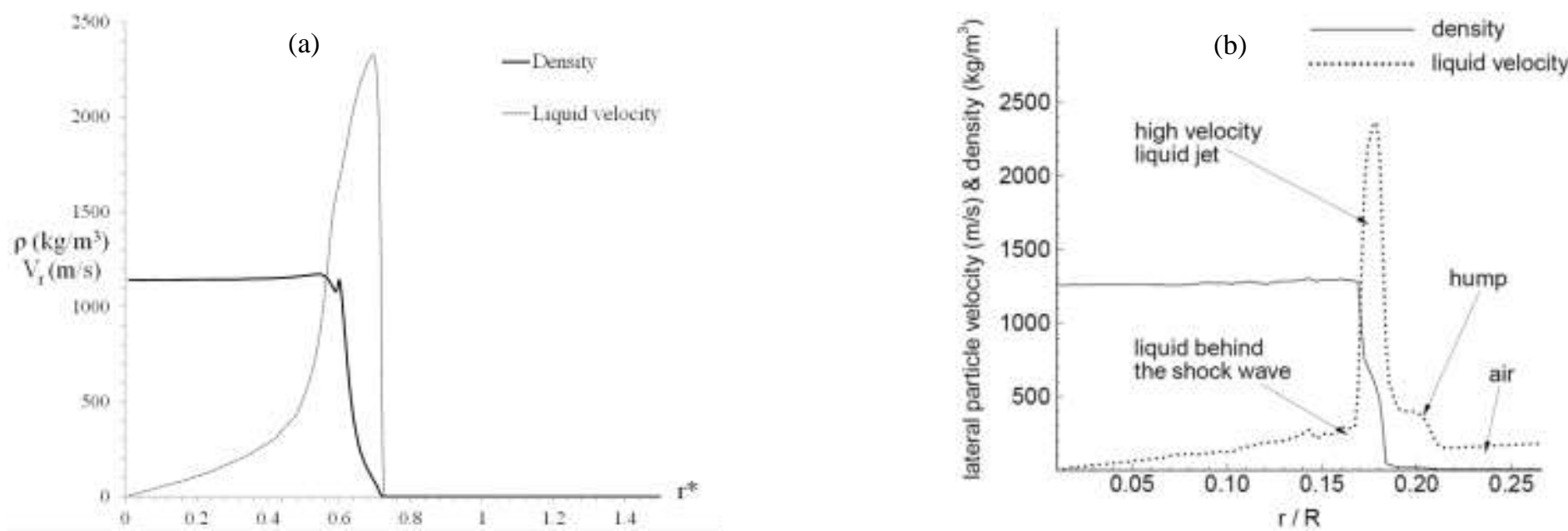

Fig. 5: Density and radial liquid velocity, (a) present work at $t^{*}=0.07$, (b) extracted from Haller [16] at $3.05 \mathrm{~ns}$ after impact.

\section{Conclusion}

In summary, the droplet impact onto a rigid substrate was modeled utilizing a compressible solver for an impact velocity of $500 \mathrm{~m} / \mathrm{s}$ and a droplet size of $200 \mu \mathrm{m}$. The results revealed the air entrapment and the appearance of the maximum pressure $(2.8 \mathrm{GPa})$ when the lateral jetting occurs at a radial velocity of $2325 \mathrm{~m} / \mathrm{s}$. Liquid water experiences about $15 \%$ increase in density. However, the density of entrapped air reaches as high as 9.61 times the undisturbed water density. The development of the compressed region and its growth inside the droplet has been shown and compared against open literature. Moreover, it is most likely that cavitation happens at the droplet periphery due to the high temperature $(771 \mathrm{~K})$. As a continuation of the present work, the phase change model can be added to the solver to study the cavitation phenomenon in more detail.

\section{Acknowledgements}

This work was funded by Consortium de Recherche et d'Innovation en Aérospatiale au Québec (CRIAQ), Natural Sciences and Engineering Research Council of Canada (NSERC), Le Fonds Québécois de la Recherche sur la Nature et les Technologies (FQRNT), Nano-Quebec, Hydro Quebec and Siemens Canada (formerly Rolls-Royce Canada).

\section{References}

[1] A. Worthington, "On the forms assumed by drops of liquids falling vertically on a horizontal plate," in Proceedings of the Royal Society of London, 1876.

[2] J. Fukai, Y. Shiiba, T. Yamamoto, O. Miyatake and D. Pouli, "Wetting effects on the spreading of a liquid droplet colliding with a flat surface: experiment and modeling," Physics of Fluids, vol. 7, no. 2, pp. 236-247, 1995.

[3] S. Chandra and C. T. Avedisian, "On the Collision of a Droplet with a Solid Surface," Proceedigs of the Royal Society, vol. 432, no. 1884, 1991.

[4] M. Pasandideh-Fard, Y. M. Qiao, S. Chandra and J. Mostaghimi, "Capillary effects during droplet impact on a solid surface," Physics of Fluids, vol. 8, no. 3, 1996.

[5] I. V. Roisman, R. Rioboo and C. Tropea, "Normal impact of a liquid drop on a dry surface: model for spreading and receding," Proceedings of the Royal Society, vol. 458, no. 2022, 2002. 
[6] V. Mehdi-Nejad, J. Mostaghimi and S. Chandra, "Air bubble entrapment under an impacting droplet," Physics offluids, vol. 15, no. 1, pp. 173-183, 2003.

[7] N. Z. Mehdizadeh, S. Chandra and J. Mostaghimi, "Formation of fingers around the edges of a drop hitting a metal plate with high velocity," Journal of Fluid Mechanics, vol. 510, pp. 353-373, 2004.

[8] T. D. Blake and J. De Conick, "The influence of solid-liquid interactions on dynamic wetting," Advances in Colloid and Interface Science, vol. 96, no. 1-3, pp. 21-36, 2002.

[9] M. Marzbali, "Numerical Analysis of High-speed Droplet Impingement on Elastic and Rigid Substrates," Concordia University, 2017.

[10] M. Marzbali, A. Dolatabadi and P. Jedrzejowski, "Fluid-Solid Interaction Modeling of Compressible Droplet Impact onto Elastic Substrates," in 21st AIAA Computational Fluid Dynamics Conference, San Diego, 2013.

[11] F. J. Heymann, "High-speed impact between a liquid drop and a solid surface," Journal of Applied Physics, vol. 40, pp. 5113-22, 1969.

[12] J. P. Dear and J. E. Field, "High-speed photography of surface geometry effects in liquid/solid impact," Journal of Applied Physics, vol. 63, pp. 1015-21, 1988.

[13] J. E. Field, J. P. Dear and J. E. Ogren, “The Effects of Target Compliance on Liquid Drop Impact," Journal of Applied Physics, vol. 65, pp. 533-40, 1989.

[14] M. C. Rochester and J. H. Brunton, "Erosion by Liquid and Solid Impact," in International Conference on Erosion by Liquid and Solid Impact (5th), Cambridge, 1979.

[15] M. B. Lesser and J. E. Field, "The Impact of Compressible Liquids,” Annual Review of Fluid Mechanics, vol. 15, pp. 97-122, 1983.

[16] K. Haller, "High-Velocity Impact of a Liquid Droplet on a Rigid Surface: The Effect of Liquid Compressibility," Swiss Federal Institure of Technology, Zurich, 1972.

[17] P. G. Tait, "Report on some of the physical properties of fresh water and sea water," Physical Chemistry, vol. 2, p. 1$17,1888$.

[18] J. U. Brackbill, D. B. Kothe and C. Zemach, “A continuum method for modelling surface tension,” vol. 100, no. 2, p. 335-354, 1992.

[19] C. Hirt and B. Nichols, "Volume of Fluid (VOF) Method for the Dynamics of Free Boundaries," vol. 39, no. 1, pp. 201-225, 1981.

[20] D. L. Youngs, “Time-Dependent Multi-Material Flow with Large Fluid Distortion,” Numerical Methods for Fluid Dynamics, 1982. 